Regular Article

\title{
Novel connection between newborn granule neurons and the hippocampal CA2 field
}

\author{
María Llorens-Martín *, Jerónimo Jurado-Arjona, Jesús Avila, Félix Hernández * \\ Centro de Biología Molecular Severo Ochoa (CSIC-UAM), c/Nicolás Cabrera 1, 28049 Madrid, Spain \\ Centro de Investigación Biomédica en Red sobre Enfermedades Neurodegenerativas (CIBERNED, ISCIII), c/Valderrebollo 5, Madrid, Spain
}

\section{A R T I C L E I N F O}

\section{Article history:}

Received 3 August 2014

Revised 12 October 2014

Accepted 23 October 2014

Available online 1 November 2014

\section{Keywords:}

Adult neurogenesis

Granule neurons

CA2

Retrovirus

Mossy fiber terminal

\begin{abstract}
A B S T R A C T
Newborn neurons are continuously added to the hippocampal dentate gyrus (DG) throughout life. Mature and immature granule neurons are believed to send their axonal projections exclusively to the hippocampal CA3 field. However, recent data point to an alternative trisynaptic circuit, involving a direct axonal projection from mature granule neurons to the CA2 field. Whether this circuit takes place only in mature granule neurons or, on the contrary, whether immature granule neurons also contribute to this novel connection is unknown. We used various retroviral vectors to show that immature granule neurons send axonal processes to and establish synaptic contacts with CA2 pyramidal neurons and that axonal growth follows a similar time course to that described for CA3 innervation. In addition, we provide experimental evidence demonstrating that the pathway connecting newborn granule neurons and the CA2 field can be modulated by physiological and deleterious stimuli.
\end{abstract}

(c) 2014 Elsevier Inc. All rights reserved.

\section{Introduction}

Newborn neurons are continuously added to the subgranular zone (SGZ) of the hippocampal dentate gyrus (DG) throughout life. Progenitor cells asymmetrically divide and give rise to transiently amplifying neuronal precursors. Immature neuroblasts actively proliferate and differentiate into mature granule neurons, going through various stages of maturation. During the differentiation of newborn neurons their dendritic tree complexity increases, and axons are projected towards the CA3 region (Zhao et al., 2006), establishing functional synapses with CA3 pyramidal neurons (Toni et al., 2008). At the end of this maturation process, newborn neurons are fully integrated in the classic trisynaptic circuit and are functionally indistinguishable from surrounding mature granule neurons (Zhao et al., 2006). However, during immaturity, newborn neurons are characterized by a lower activation threshold (Schmidt-Hieber et al., 2004), and adult hippocampal neurogenesis (AHN) is involved in hippocampal-dependent learning, being crucial for pattern separation (Clelland et al., 2009; Nakashiba et al., 2012). AHN is a tightly regulated process modulated by numerous external stimuli.

It has been widely demonstrated that the classic hippocampal trisynaptic circuit (Enthorrinal Cortex ((EC))-DG-CA3-CA1) is one of

\footnotetext{
* Corresponding authors at: Centro de Biología Molecular "Severo Ochoa”, Universida Autónoma de Madrid, c/Nicolás Cabrera 1, 28049 Madrid, Spain. Fax: +34911964420. E-mail addresses: m.llorens@csic.es (M. Llorens-Martín), fhernandez@cbm.uam.es (F. Hernández).
}

the most important networks involved in memory and learning processes. However, recent data point to the existence of an alternative trisynaptic circuit (Kohara et al., 2014) that involves the connection between mature granule neurons and CA2 pyramidal neurons. This alternative circuit is crucial for contextual and social memory (Hitti and Siegelbaum, 2014; Wintzer et al., 2014). However, whether newborn granule neurons contribute to this connection remains unknown. Here we analyzed the potential contribution and time course of the connections between newborn granule neurons and the hippocampal CA2 region. To this end, we used various retroviral vectors (encoding for either GFP or Synaptophysin-GFP), and specific markers for the axons of newborn neurons (3R-Tau) and for the CA2 field (RGS14 and PCP4) (Antonio et al., 2014; Evans et al., 2014). In addition, we show how both deleterious and neuroprotective stimuli modulate these connections.

\section{Material and methods}

Animals

Six-week-old female C57BL/6JRcc mice were obtained from Harlan Laboratories. Animals were housed in a specific pathogen-free colony facility, in accordance with European Community Guidelines (directive 86/609/EEC), and handled following European and local animal care protocols. Animal experiments received the approval of the CBMSO's Ethics Committee. Five mice per experimental condition and time point were used in all the experiments. 


\section{Physical exercise protocol}

Animals were habituated to the treadmill apparatus for 15 min (Cibertec, Madrid, Spain). The running speed was $0.2 \mathrm{~m} / \mathrm{s}$. Animals ran 7 days per week for $40 \mathrm{~min} /$ day over 2 weeks. Sedentary animals remained on the treadmill without running for the same period of time. Animals were eight weeks old at the beginning of the exercise protocol.

\section{Treatment with lipopolysaccharide from Escherichia coli (LPS)}

LPS was chronically administered during 8 weeks subcutaneously via Alzet ${ }^{\circledR}$ osmotic pumps (model 1004) (Durect Corporation, Cupertino, California). To obtain a continuous LPS delivery of $300 \mu \mathrm{g} / \mathrm{kg} /$ day for 8 weeks, pumps were filled with a solution of LPS (Sigma, from E. coli 055:B5, St. Louis, MO) diluted in 0.1 M PBS. Pumps were replaced by new ones 4 weeks after the beginning of the treatment. Control mice carried a PBS-containing osmotic pump and were subjected to the same experimental manipulations than LPS-treated mice. Animals were eight weeks old when they were operated for the first time.

\section{Retroviral stock preparation}

We used two retroviral stocks, which varied in the genes encoded: CAG-GFP encoding for GFP (Zhao et al., 2006) and Synaptophysin-GFP (Syn-GFP) for GFP fused with Synaptophysin (Kelsch et al., 2009). Since both retroviruses used are engineered to be replication incompetent, only dividing cells at the time of surgery can be infected (Kelsch et al., 2008; Zhao et al., 2006). On the one hand, GFP-expressing retroviruses were used to allow the visualization of the whole morphology (including the MFT) of the newborn neurons. On the other hand, the Syn-GFP retrovirus allowed Syn ${ }^{+}$presynaptic cluster visualization. Moreover, anti-GFP immunohistochemistry allowed axon and whole mossy fiber presynaptic terminal visualization when using this type of retrovirus (Kelsch et al., 2009). The plasmids used for the production of the GFP-expressing retrovirus were a generous gift from Prof. Fred H. Gage (Salk Institute), while those used to produce the Syn-GFP retrovirus were kindly provided by Prof. Carlos Lois (University of Massachusetts). Retroviral stocks were concentrated to working titers of $1 \times 10^{7} \mathrm{pfu} / \mathrm{ml}$ by ultracentrifugation (Zhao et al., 2006).

The time course of newborn neuron-CA2 synaptic contact establishment was studied by using the GFP-expressing retrovirus, whereas the modulation by LPS was analyzed by using the Syn-GFP retrovirus. Although the presence of Synaptophysin at the synaptic bouton is not a measure of functionality, it further confirms their existence and possibility of function.

\section{Stereotaxic surgery}

Mice were anesthetized with Isoflouran and placed in a stereotaxic frame. Coordinates $(\mathrm{mm})$ relative to the bregma in the anteroposterior, mediolateral, and dorsoventral planes were as follows: DG [-2.0, 1.4, 2.2]. $2 \mu \mathrm{l} / \mathrm{DG}$ of virus solution was infused at $0.2 \mu \mathrm{l} / \mathrm{min}$ via a glass micropipette. Animals were 8 weeks old at the moment of retroviral injections. Five mice per experimental condition and time point were used for retroviral labeling experiments.

\section{Sacrifice}

Mice were fully anesthetized by means of an intraperitoneal injection of pentobarbital and transcardially perfused with saline followed by $4 \%$ paraformaldehyde in phosphate buffer. Brains were removed and post-fixed overnight in the same fixative.

\section{Immunohistochemistry}

Sagittal brain sections were obtained on a Leica VT1200S vibratome (50- $\mu \mathrm{m}$ thick sections). Immunohistochemistry was performed as described previously (Llorens-Martin et al., 2013). For all the immunohistochemical analysis, series of brain slices were made up randomly of one section from every 9 th. One series, containing 8-10 sections, was used for each immunohistochemical determination. The following primary antibodies were used: rabbit anti-GFP (Molecular Probes (Invitrogen), 1:1000); mouse anti-RGS14 (Neuromab, 1: 1,000); mouse anti-3RTau (EMD Millipore, 1:500); and rabbit anti-PCP4 (Sigma-Aldrich, 1:500). The following secondary Alexa-conjugated antibodies were used at a final concentration of 1:1,000: Donkey Alexa 555-conjugated antirabbit (Life Technologies); Donkey Alexa 488-conjugated anti-mouse (Life Technologies); Donkey Alexa 488-conjugated anti-rabbit, (Life Technologies); Donkey Alexa 647-conjugated anti-rabbit (Life Technologies). All sections were counterstained with DAPI (Calbiochem, 1:5000). In the case of 3RTau immunohistochemistry, an antigen retrieval step was required to visualize the axons of newborn neurons (Llorens-Martin et al., 2012).

\section{Mossy fiber terminal area measurement}

Ten stacks of images per mouse and anatomical region were obtained in a LSM710 Zeiss confocal microscope. Stacks of images were randomly obtained among the different sections composing the series $(63 \times$ Oil immersion objective, xy dimensions: $60 \mu \mathrm{m}$ ) (Z-axis interval: $0.13 \mu \mathrm{m})$. The area of each individual mossy fiber terminal (MFT) contained in the images was measured in both the CA2 and CA3 regions. Data averaged on the five mice are presented in the graphs and have been used to perform statistics. A minimum of 500 terminals per experimental condition and time point were measured. GFP Z-projections were obtained and the area of each terminal bouton was measured manually in ImageJ as previously described (Zhao et al., 2006). All the different measurements and analysis were performed by the same investigator blinded to experimental condition.

\section{Synaptophysin ${ }^{+}$area of the mossy fiber terminal quantification}

Ten stacks of images per mouse and anatomical region were obtained in a LSM710 Zeiss confocal microscope. Stacks of images were randomly obtained among the different sections composing the series. The area of

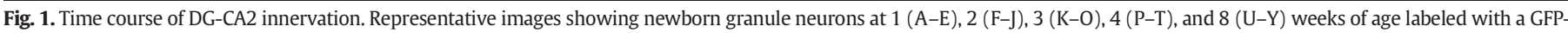

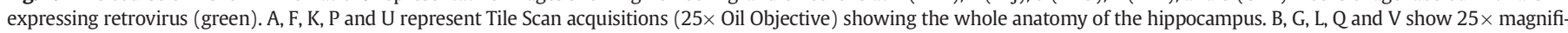

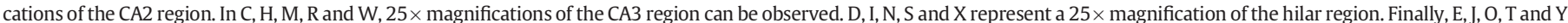

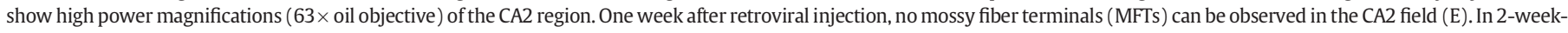

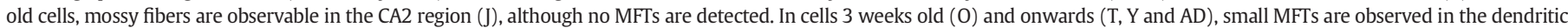

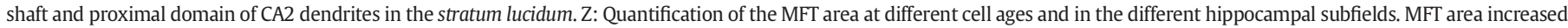

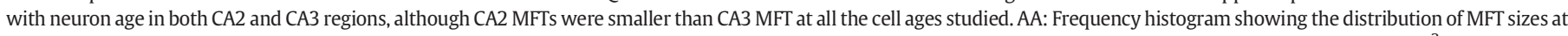

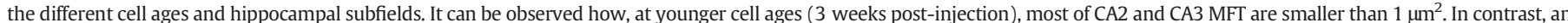

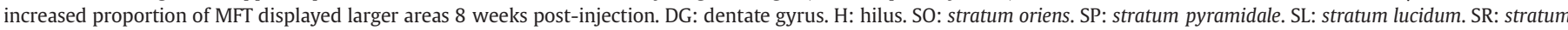

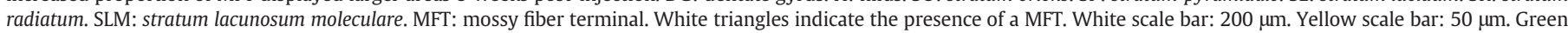
scale bar: $20 \mu \mathrm{m}$. 
each individual mossy fiber terminal MFT was measured in the GFP channel of stack Z-projection images. Subsequently, Synaptophysin channel Z-projections were thresholded (Lower value: 78; Upper value: 255) and the $\mathrm{Syn}^{+}$area of each individual MFT was measured manually in Image J. The percentage of $\mathrm{Syn}^{+}$area was calculated for each individual MFT and represented in Fig. 2. In the 4-channel images of the figures, Synaptophysin channel is represented by a Grey channel pseudocolor. Data averaged on the five mice are presented in the graphs and have been used to perform statistics. A minimum of 500 terminals per experimental condition were individually measured. All the different measurements and analysis were performed by the same investigator blinded to experimental condition.

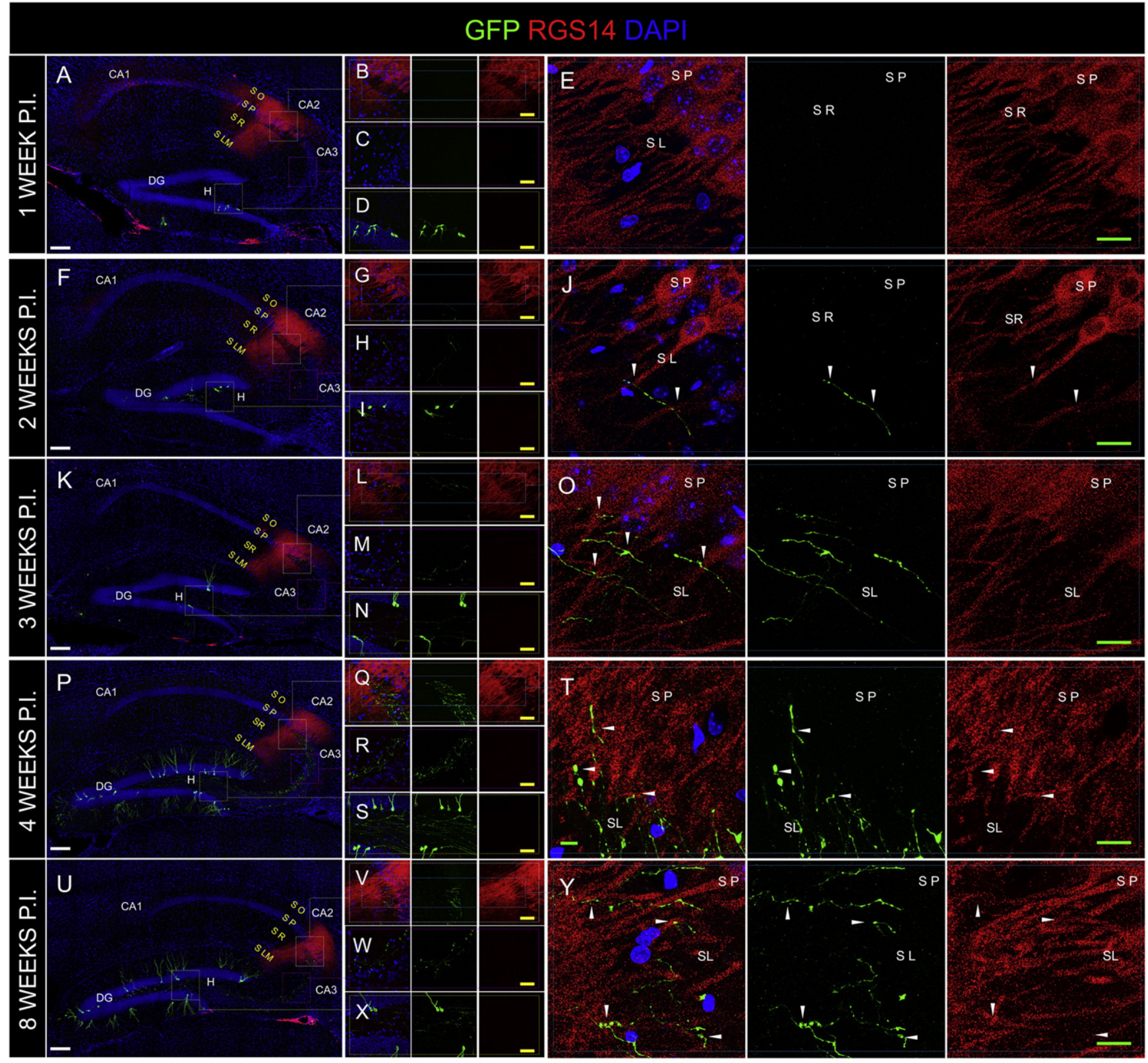

\section{Z}

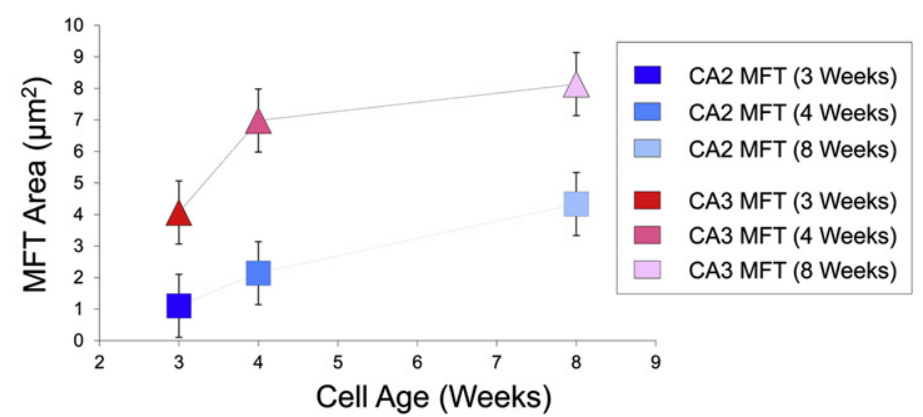

AA

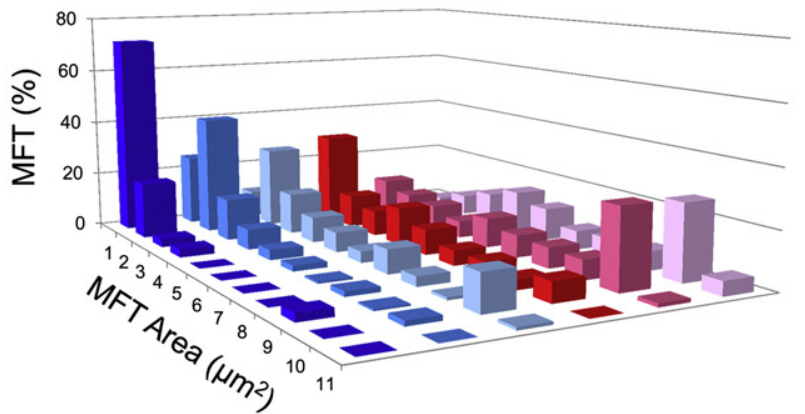


Measurement of $3 R-\mathrm{Tau}^{+}$area in the $\mathrm{CA} 2$ and $\mathrm{CA} 3$ regions

Three confocal stacks of images per animal were obtained in a LSM710 Zeiss confocal microscope (63× Oil immersion objective, xy dimensions: $130 \mu \mathrm{m}$ ) (Z-axis interval: $0.5 \mu \mathrm{m}$ ). Stacks of images were randomly obtained among the different sections composing the series. By using PCP4 staining for guidance, a region of interest (either in the $\mathrm{CA} 2\left(\mathrm{PCP}^{+}\right)$or $\mathrm{CA} 3\left(\mathrm{PCP}^{-}\right)$regions) was first delineated within the stratum lucidum (SL) and then this area was measured in the
PCP4 channel of Z-projection images. Subsequently, 3R-Tau channel Z-projections were thresholded (Lower value: 120; Upper value: 255 ) and the 3R-Tau ${ }^{+}$area was measured in ImageJ. The percentage of $3 \mathrm{R}-\mathrm{Tau}^{+}$area was normalized by dividing the $3 \mathrm{R}-\mathrm{Tau}^{+}$area obtained by the total area of the region measured in the PCP4 channel. Data averaged on the five mice are presented in the graphs and have been used to perform statistics. All the different measurements and analyses were performed by the same investigator blinded to experimental condition.
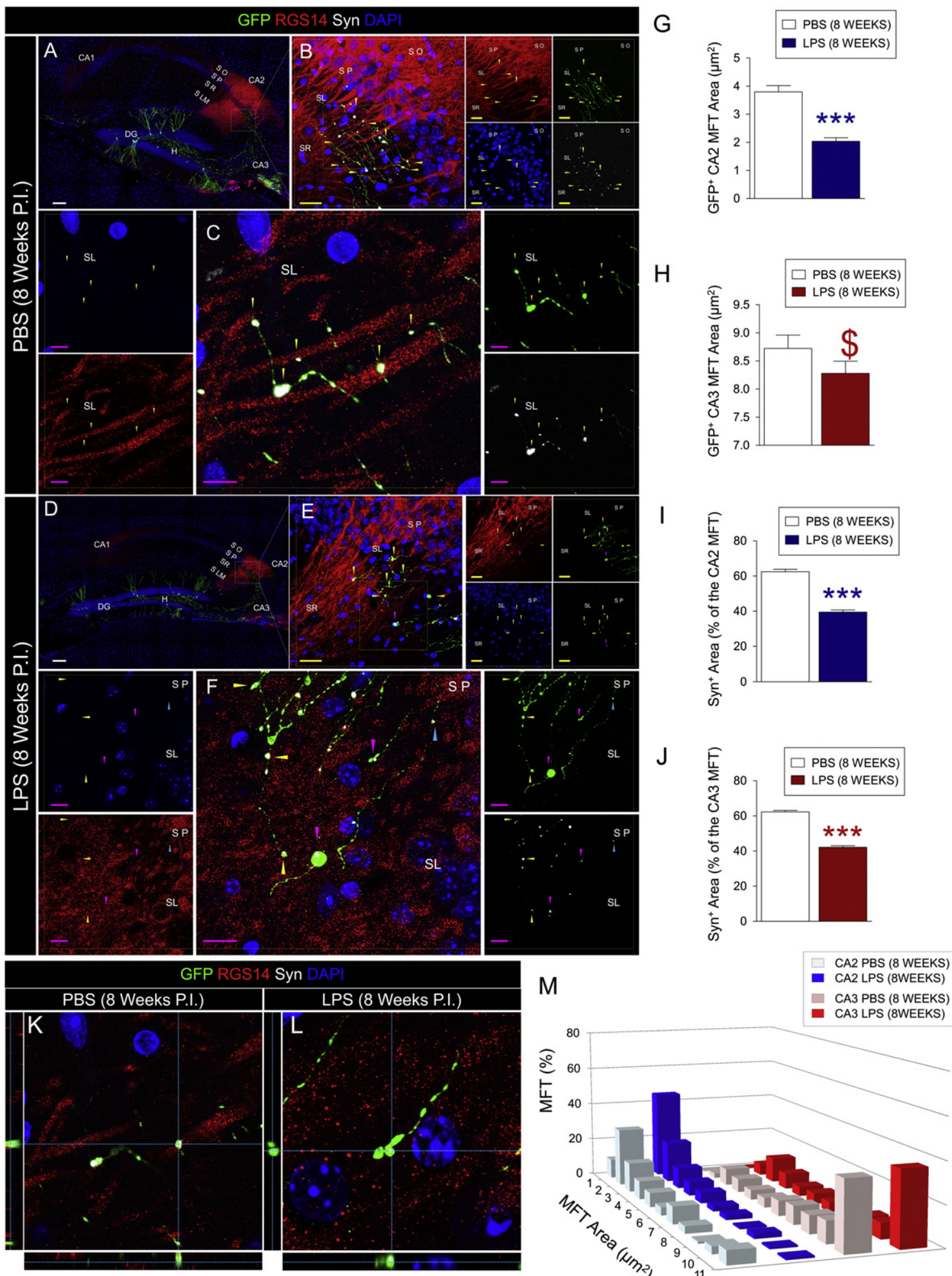

M

- CA2 LPS (8WEEKS)

CA3 LPS (8WEEKS)

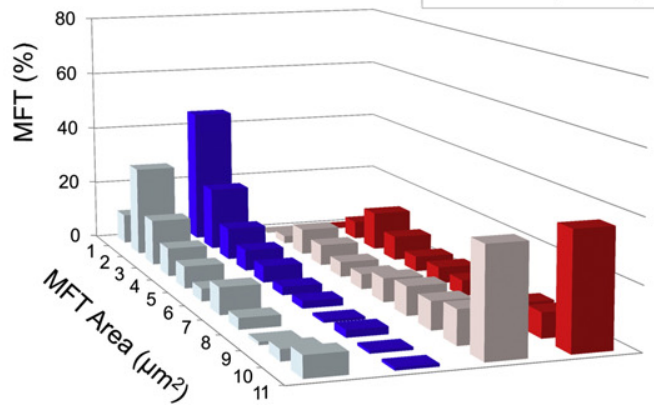




\section{Cell counting}

The density of 3R-Tau ${ }^{+}$immature granule neurons was calculated using the physical dissector method adapted for confocal microscopy (Llorens-Martin et al., 2006) using a LSM710 Zeiss confocal microscope (63× Oil immersion objective). Briefly, 5 confocal stacks of images per mouse, corresponding to the subgranular zone and granule layer of the dentate gyrus, were obtained. Stacks of images were randomly obtained among the different sections composing the series. The number of cells counted was divided by the area of the subgranular zone measured in each stack. The density of 3R-Tau ${ }^{+}$cells (number of cells $/ \mu m^{2}$ ) is represented in Fig. 3. Data averaged on the five mice are presented in the graphs and have been used to perform statistics. All the different measurements and analysis were performed by the same investigator blinded to experimental condition.

\section{Statistical analysis}

All statistics were analyzed using the SPSS 17.0.1 software (SPSS, 1989; Apache Software Foundation). The Kolmogorov-Smirnov test was used to test the normality of the sample distribution. A nonparametric test (Mann-Whitney U test) was applied in those cases in which normality could not be assumed. In case of normal sample distribution, a two independent samples Student t-test was applied.

\section{Results}

Here we sought to determine whether there are direct connections between newborn granule and CA2 pyramidal neurons. For this purpose, we injected two types of retrovirus in order to analyze the time course, anatomical distribution, and morphology of the mossy fiber terminals (MFTs) of newborn granule neurons in the CA2 field.

\section{Time course of DG-CA2 connections}

To study the establishment of synaptic connections between newborn neurons and CA2 pyramidal neurons, we followed the time course of the former (Fig. 1). To that aim, we stereotaxically injected GFP-expressing retroviruses into the hippocampus of wild type (wt) mice and sacrificed the animals at a range of times post-injection $(1,2,3,4,8$, or 10 weeks). One week after retroviral injections, no MFTs were observed in the CA2 or CA3 fields (Figs. 1A-E). Two weeks after retroviral injection, axonal processes were detected in the CA2 region for the first time (Figs. 1F-J). Accordingly, innervation towards the CA3 zone was prominent. No MFTs were observed in the CA2 region at this time point. At 3 weeks post-injection (Figs. $1 \mathrm{~K}-\mathrm{O}$ ) and onwards (Figs. 1P-Y), MFTs established contacts with the apical dendrites of CA2 pyramidal neurons. Most of these contacts were observed in the stratum lucidum (SL), although few perisomatic contacts were also seen. Consistent with CA3 MFTs, the area of CA2 MFTs increased with neuron age. However, CA2 MFTs displayed a smaller area as compared to that of CA3 MFTs (Fig. 1Z), as previously described for the MFTs of mature granule neurons (Kohara et al., 2014). CA2 MFTs belonging to 3- and 4-week-old neurons displayed a fusiform morphology, whereas those belonging to 8-week-old neurons displayed a complex morphology with multi-terminal ends (Fig. 1Y). In Fig. 1 AA, the distribution of MFT sizes at the different cell ages and hippocampal subfields is presented. It is important to note that, at younger cell ages ( 3 weeks post-injection), most of both CA2 and CA3 MFTs displayed areas smaller than $1 \mu \mathrm{m}^{2}$. In contrast, most of the mature granule neurons ( 8 weeks of cell age) MFT had larger areas in both regions. Interestingly, this increase in the MFT area can be observed at younger cell ages in the CA3 field (4 weeks post-injection) than in the CA2 region ( 8 weeks post-injection).

Establishment of synapses between DG granule neurons and the CA2 region

To further demonstrate that newborn neurons establish connections with CA2 pyramidal neurons, we used a retrovirus encoding Synaptophysin and GFP (Syn-GFP retrovirus). Although this is not the direct measure of functionality, it can be considered a further confirmation of their existence and possibility of function. Newborn neurons, which are considered to be fully mature at 8 weeks of age, established functional synapses with CA3 pyramidal neurons (Toni et al., 2008; Zhao et al., 2006). Eight weeks after retroviral injection, CA2 MFTs were Synaptophysin ${ }^{+}$, thus indicating the existence of synaptic contacts between 8-week-old newborn neurons and CA2 pyramidal neurons (Figs. 2A-C). In contrast to the presence of thorny excrescences on the dendrites of CA3 pyramidal neurons (Fitch et al., 1989), the dendrites of CA2 pyramidal neurons lacked complex spines, and synaptic contacts occurred in the proximal domain of the dendritic shaft located in the SL, although a few contacts were observed in the stratum pyramidale (SP). We have quantified the area of the MFT occupied by Synaptophysin ${ }^{+}$clusters under physiological conditions in both the CA2 and the CA3 regions, and surprisingly, we have obtained that the average $\mathrm{Syn}^{+}$area was around $61 \%$ of the total MFT in both regions (Figs. 2I,J), despite the different size and nature of the synaptic contacts established in both regions.

\section{CA2 MFTs are affected by inflammation}

Inflammation negatively regulates hippocampal connectivity and functionality (Maggio et al., 2013; Monje et al., 2003). With the aim to study whether DG-CA2 connectivity is also disrupted by inflammation, we peripherally administered the general pro-inflammatory agent LPS, known to produce brain inflammation in a wide variety of conditions (Cunningham et al., 2005). Under physiological conditions, Syn ${ }^{+}$ clusters are homogenously distributed among the MFT, and a single round and large Syn $^{+}$cluster can be visualized in each MFT (Fig. 2C). In contrast, LPS administration caused a severe alteration of CA2 MFT morphology (Figs. 2D-F,K-L). In LPS-treated mice, Syn was observed to be distributed irregularly within the MFTs (a high abundance of

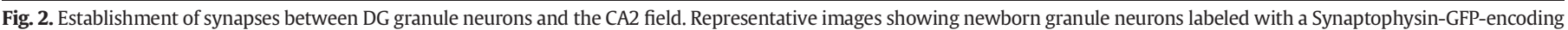

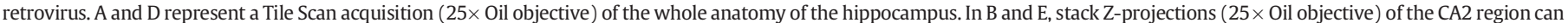

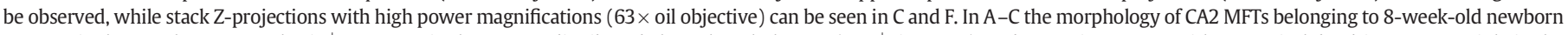

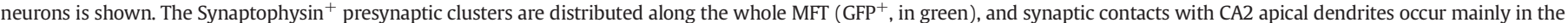

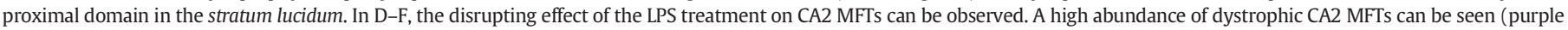

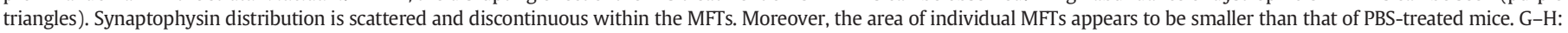

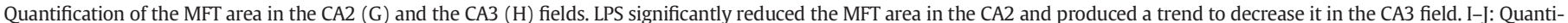

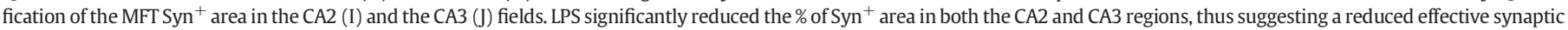

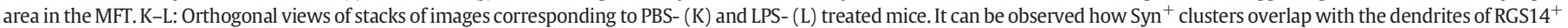

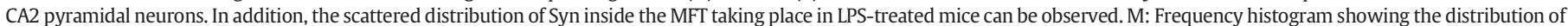

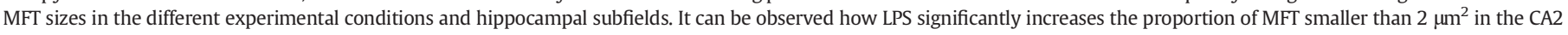

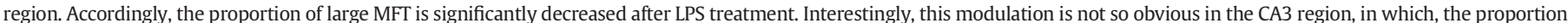

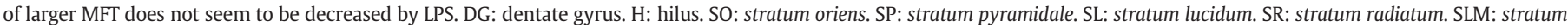

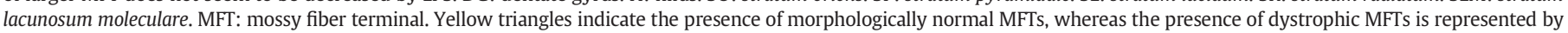
purple triangles. White scale bar: $100 \mu \mathrm{m}$. Yellow scale bar: $50 \mu \mathrm{m}$. Purple scale bar: $10 \mu \mathrm{m}$. $\left({ }^{* * *} \mathrm{p}<0.001\right)\left({ }^{\$} 0.05<\mathrm{p}<0.1\right)$. 
pale scattered dots of synaptophysin appear inside the MFTs) (purple triangles). In addition, we have quantified both the area of the MFT and the area occupied by $\mathrm{Syn}^{+}$clusters after LPS administration. Figs. 2G and H show how LPS provoked a reduction of the MFT area in the CA2 region (Mann-Whitney $U \mathrm{p}<0.001, \mathrm{n}=5$ ) and a trend to reduce it in the CA3 field (Mann-Whitney $U \mathrm{p}=0.07, \mathrm{n}=5$ ). In addition, LPS significantly reduced the area of the MFT occupied by Syn $^{+}$clusters in both CA2 (Student t-test, $\mathrm{p}<0.001, \mathrm{n}=5$ ) and CA3 (Student t-test, $\mathrm{p}<0.001, \mathrm{n}=5$ ) regions (Figs. 2I and J respectively). In Fig. 2M, it can be observed the frequency histogram showing the distribution of MFT sizes in the different experimental conditions and hippocampal subfields. As shown in the graph, LPS drastically increased the proportion of small MFT (smaller than $2 \mu \mathrm{m}^{2}$ ) in the CA2 region. In contrast, the proportion of large CA2 MFT is decreased after LPS administration. Interestingly, such an outstanding modulation cannot be observed in the CA3 region, in which, the proportion of larger MFT does not seem to be decreased by LPS (Fig. 2M), although the average MFT area shows a trend to be decreased (Fig. 2H).

\section{The DG-CA2 connection can be modulated physiologically}

We have used the marker of the axons of newborn neurons 3R-Tau, in order to label the newborn neuron mossy fiber bundle (LlorensMartin et al., 2012). Fig. 3 shows representative images of the specific markers for the axons of newborn neurons (3R-Tau) and CA2 pyramidal neurons (PCP4). In Fig. 3A,C, representative images of sedentary control mice are shown, whereas Fig. 3B,D shows representative images of exercised mice. In addition to the classically described innervations of CA3 by immature neurons, it is possible to observe how $3 \mathrm{R}-\mathrm{Tau}^{+}$immature neurons also innervated the SL of the CA2 region. On the basis of the temporal pattern of 3R-Tau expression (1-4 weeks of cell age (LlorensMartin et al., 2012)), MFTs showed a reduced size and complexity. We have quantified 3R-Tau expression in both the CA2 and CA3 fields after physical exercise, and we have obtained a prominent increase in 3R-Tau staining in both regions (Fig. 3E) (Student t-test, $\mathrm{p}=0.016$ and $\mathrm{p}=0.049$ respectively, $\mathrm{n}=5$ ) as compared to sedentary control mice. It has been previously described that physical exercise increases 3R-Tau staining in the CA3 region (Llorens-Martin et al., 2012), and here we have reported a similar effect for the CA2 field (Fig. 3B,D). To the best of our knowledge, this is the first evidence of the physiological regulation of DG-CA2 connections.

In order to normalize the effects of exercise on immature neuron MFT, we have quantified the total number of 3R-Tau ${ }^{+}$immature neurons present in the DG. As shown in Fig. 3F, physical exercise significantly increased the number of 3R-Tau ${ }^{+}$immature neurons in this region (Student t-test, $\mathrm{p}=0.019, \mathrm{n}=5$ ). The net increase in neurogenesis in the DG after physical exercise $(38.6 \%)$ could account for the axonal effects observed in the CA3 region ( $49.2 \%$ of increase in 3R-Tau staining), but it is negligible compared to the increase in newborn neuron innervation towards the CA2 field (316.6\%). This may suggest the existence of specific stimulatory effects on DG-CA2 connections between immature granule and CA2 pyramidal neurons. An alternative explanation could be that the same net increase in the number presynaptic boutons has a greater impact in the CA2 than in the CA3 field, due to the lower basal innervation of the first by immature, $3 r-\mathrm{Tau}^{+}$neurons.

\section{Discussion}

In the classical view of the hippocampal trisynaptic circuit, information from the EC is transmitted sequentially to the DG, CA3 and then to CA1, thus forming the main efferent information pathway of the hippocampus (van Strien et al., 2009). Recently, a novel alternative trisynaptic circuit involving the CA2 region has been described (Kohara et al., 2014). The CA2 region is characterized by large pyramidal neurons lacking thorny excrescences on their apical dendrites. A widely accepted definition of the CA2 region also included the absence of mossy fiber innervation (de Nó, 1934; van Strien et al., 2009) and a prominent innervation from the supramammillary nucleus (Haglund et al., 1984). However, recent data obtained by novel optogenetic and electrophysiological tools have revealed unique features of this alternative circuit which is essential for social and contextual learning (Hitti and Siegelbaum, 2014; Kohara et al., 2014; Wintzer et al., 2014). It is possible that the singularity of this role is determined by the unusual and profuse connections received by the CA2 region. These connections include direct afferents from the EC (layers II and III) as an intermediate relay in the temporoammonic monosynaptic pathway (EC-CA1) (Chevaleyre and Siegelbaum, 2010), from the CA3 field (Zhao et al., 2007), and from mature granule neurons (Kohara et al., 2014), the latter being received from small MFT synaptic boutons (in contrast to the prominent
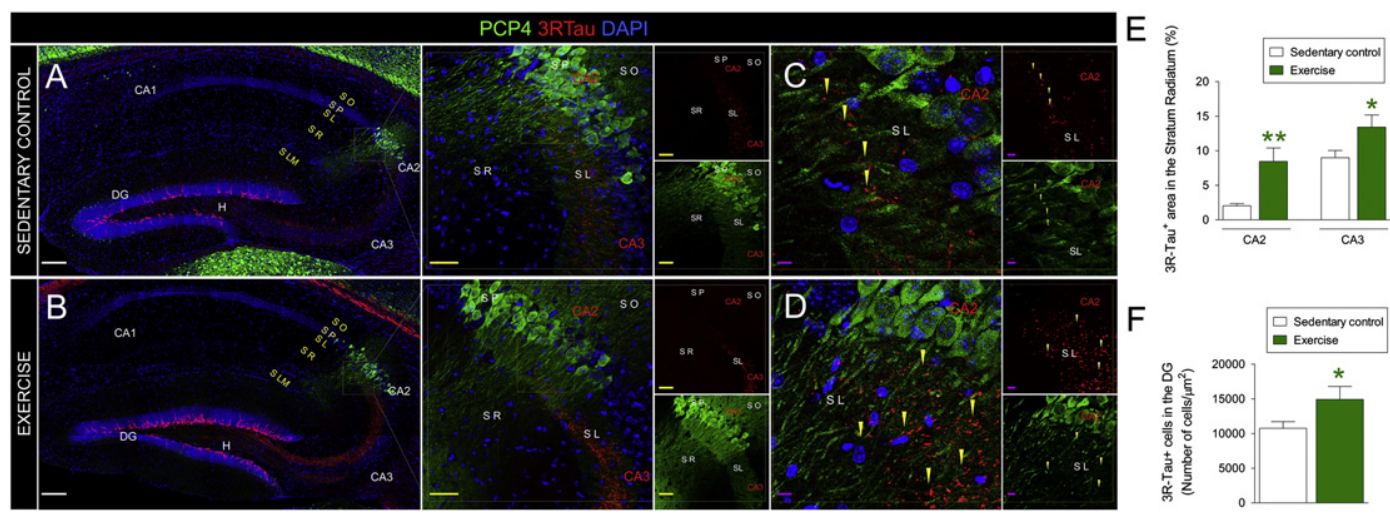

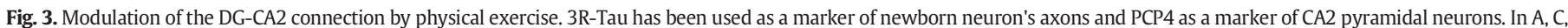

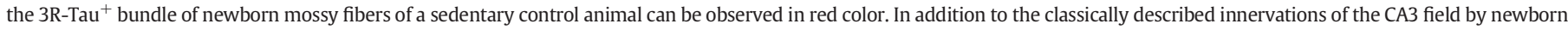

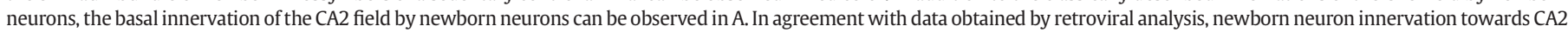

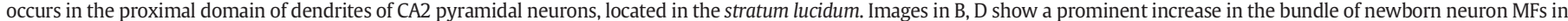

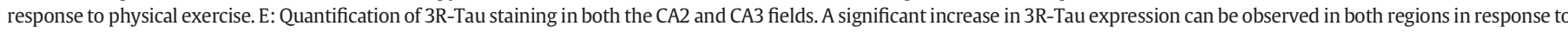

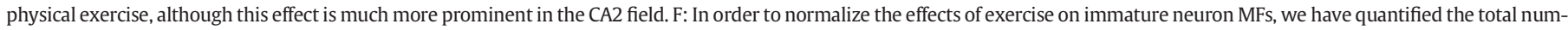

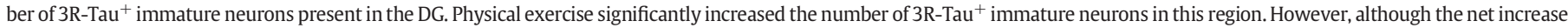

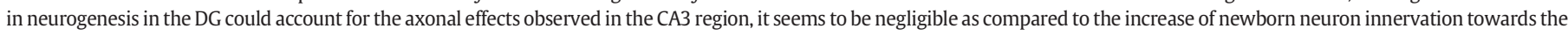

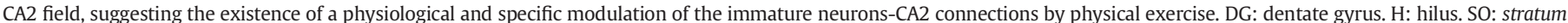

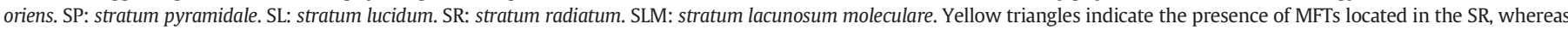

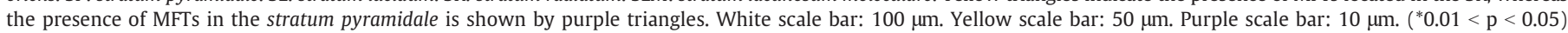
$\left({ }^{* *} 0.001<\mathrm{p}<0.01\right)$ 
terminals observable in the CA3 region) located in the SR (Kohara et al., 2014). Proximal synapses on dendrites generally have a greater effect on neuronal activity than distal ones (Andersen, 2007). Of particular interest is the observation that innervation from the DG takes place in the proximal part of the apical dendrite of CA2 pyramidal neurons, whereas CA3 innervation occurs in the distal part of these neurons, resulting in a lower synaptic strength than the DG-CA2 connection (Kohara et al., 2014). In fact, the DG-CA2 connection is stronger than some of the other connections in the cortico-hippocampal network (Kohara et al., 2014). Thus the question as to whether newborn neurons contribute to this connection is highly relevant, especially when taking into account that both the CA2 region and AHN are implicated in pattern separation. In fact, recent data point to remarkable functional similarities between the DG and CA2 region in detecting and encoding changes in patterns of similar inputs (Leutgeb et al., 2007; Wintzer et al., 2014). Not surprisingly, these two structures share a high degree of inhibitory tone (Mercer et al., 2007) and receive inputs from the supramammillary nucleus (Haglund et al., 1984), a region that plays a determinant role in reactions to novelty (Ito et al., 2009). It has been proposed that the DG and CA2 region are essential for separating the novel from the familiar features of a context (Schmidt et al., 2012). It is likely that the connections between newborn neurons play a crucial role in modulating the discriminative capacity of the CA2 region. Here we demonstrate that the temporal pattern of innervation of the CA2 region by newborn granule neurons follows the time course of that of CA3 region (Zhao et al., 2006), although a slight delay can be observed in the case of CA2 innervation as compared to that of CA3. CA2 MFTs of newborn neurons are found at around 3 weeks of cell age, coincident with the period of highest electrophysiological plasticity of newborn granule neurons (Bischofberger, 2007). However, the data obtained here suggest that the maturation of the MFT follows a different time course in the CA2 and the CA3 fields. In fact, CA3 MFT area shows a rapid increase between the third and the fourth week post-injection, whereas such an increase only takes place between the fourth and the eighth week in the case of CA2 MFT.

In addition, a crucial question is whether the subpopulation of newborn neurons is segregated in terms of the projection to the CA3 or CA2 regions. Similar to what has been described for the subventricular zone (SVZ), in which distinct subpopulations of interneurons are generated from various proliferative clusters (Lledo et al., 2008), some newborn neurons generated in the DG may project their axons exclusively to the CA3 region while others project to CA2. Alternatively, each newborn neuron may project its axon first to the CA3 field, and the axonal projection may extend to reach CA2 field.

We have demonstrated here that inflammation threatens the integrity of DG-CA2 connections, reducing not only the total area of the MFTs but also the percentage of area occupied by Synaptophysin ${ }^{+}$clusters, thus presumably resulting in a smaller synaptic strength. This fact deserves further attention, in order to determine the selective involvement of this specific disruption in behavioral alterations mediated by inflammation. Inflammation is known to produce severe memory impairments, and given the crucial role in controlling certain types of hippocampal-related memory played by the connection between the DG and the CA2 field, it seems reasonable to consider that the specific disruption of the connections between newborn granule neurons and the CA2 field may be related to the specific impairment of certain types of memory caused by inflammation.

Importantly, we have also demonstrated that a physiological, neuroprotective stimulus such as physical exercise is able to reinforce the connection between newborn granule neurons and the CA2 field. By using the marker of newborn granule neuron axons 3R-Tau, we have demonstrated here an increased innervation of both the CA2 and the CA3 fields after physical exercise. However, the increase in 3R-Tau staining is much more prominent in the case of the CA2 field than in the CA3 region. Taken together, these data suggest the existence of a hitherto unknown mechanism of hippocampal plasticity which might be relevant for hippocampal functioning.
The combination of behavioral and genetic tools aimed to selectively block the connections between newborn neurons and the CA2 field are essential to unveil the physiological significance of the previously described modulations. Although electrophysiological or optogenetics approaches will be needed to demonstrate the newborn granule neuronCA2 functional connectivity and whether these connections could affect hippocampal behavior, these experiments provide further confirmation of their existence and possibility of function. Indeed, our data suggest that both neuroprotective and deleterious stimuli modulate this connection simultaneously to both AHN and hippocampal-dependent memory regulation (Belarbi et al., 2012; Cassilhas et al., 2012). Our findings reveal a novel mechanism of hippocampal plasticity, which may have important functional and behavioral consequences.

\section{Conflicts of interest}

The authors declare no conflict of interest.

\section{Acknowledgments}

This study was funded by grants from the Spanish Ministry of Health (SAF 2006-02424, BFU-2008-03980, BFU-2010-21507), the Comunidad de Madrid (SAL/0202/2006), the Centro de Investigación Biomédica en Red sobre Enfermedades Neurodegenerativas (CIBERNED, ISCIII) (CB401), and the Fundación R. Areces.

The authors thank E. García for the help in producing retroviral vectors; N. de la Torre for the help with manuscript edition; F.H. Gage for providing the plasmids used for the production of GFP-expressing retroviruses; and C. Lois for providing the plasmids used for the production of the PSD95-GFP virus.

\section{References}

Andersen, P., 2007. The Hippocampus Book. Oxford University Press.

Antonio, A.S., Liban, K., Ikrar, T., Tsyganovskiy, E., Xu, X., 2014. Distinct physiological and developmental properties of hippocampal CA2 subfield revealed using antiPurkinje cell protein 4 (PCP4) immunostaining. J. Comp. Neurol. 522 (6), 1333-1354.

Belarbi, K., Arellano, C., Ferguson, R., Jopson, T., Rosi, S., 2012. Chronic neuroinflammation impacts the recruitment of adult-born neurons into behaviorally relevant hippocampal networks. Brain Behav. Immun. 26, 18-23.

Bischofberger, J., 2007. Young and excitable: new neurons in memory networks. Nat. Neurosci. 10, 273-275.

Cassilhas, R.C., Lee, K.S., Fernandes, J., Oliveira, M.G., Tufik, S., Meeusen, R., de Mello, M.T., 2012. Spatial memory is improved by aerobic and resistance exercise through divergent molecular mechanisms. Neuroscience 202, 309-317.

Chevaleyre, V., Siegelbaum, S.A., 2010. Strong CA2 pyramidal neuron synapses define a powerful disynaptic cortico-hippocampal loop. Neuron 66, 560-572.

Clelland, C.D., Choi, M., Romberg, C., Clemenson Jr., G.D., Fragniere, A., Tyers, P., Jessberger, S., Saksida, L.M., Barker, R.A., Gage, F.H., Bussey, T.J., 2009. A functional role for adult hippocampal neurogenesis in spatial pattern separation. Science 325, 210-213.

Cunningham, C., Wilcockson, D.C., Campion, S., Lunnon, K., Perry, V.H., 2005. Central and systemic endotoxin challenges exacerbate the local inflammatory response and increase neuronal death during chronic neurodegeneration. J. Neurosci. 25, 9275-9284.

de Nó, R.L., 1934. Studies on the structure of the cerebral cortex. II. Continuation of the study of the ammonic system. J. Psychol. Neurol. 46, 64 (Lpz).

Evans, P.R., Lee, S.E., Smith, Y., Hepler, J.R., 2014. Postnatal developmental expression of regulator of G protein signaling 14 (RGS14) in the mouse brain. J. Comp. Neurol. 522, 186-203.

Fitch, J.M., Juraska, J.M., Washington, L.W., 1989. The dendritic morphology of pyramidal neurons in the rat hippocampal CA3 area. I. Cell types. Brain Res. 479, 105-114.

Haglund, L., Swanson, L.W., Kohler, C., 1984. The projection of the supramammillary nucleus to the hippocampal formation: an immunohistochemical and anterograde transport study with the lectin PHA-L in the rat. J. Comp. Neurol. 229, 171-185.

Hitti, F.L., Siegelbaum, S.A., 2014. The hippocampal CA2 region is essential for social memory. Nature 508 (7494), 88-92.

Ito, M., Shirao, T., Doya, K., Sekino, Y., 2009. Three-dimensional distribution of Fos-positive neurons in the supramammillary nucleus of the rat exposed to novel environment. Neurosci. Res. 64, 397-402.

Kelsch, W., Lin, C.W., Lois, C., 2008. Sequential development of synapses in dendritic domains during adult neurogenesis. Proc. Natl. Acad. Sci. U. S. A. 105, 16803-16808.

Kelsch, W., Lin, C.W., Mosley, C.P., Lois, C., 2009. A critical period for activity-dependent synaptic development during olfactory bulb adult neurogenesis. J. Neurosci. 29, $11852-11858$.

Kohara, K., Pignatelli, M., Rivest, A.J., Jung, H.Y., Kitamura, T., Suh, J., Frank, D., Kajikawa, K., Mise, N., Obata, Y., Wickersham, I.R., Tonegawa, S., 2014. Cell type-specific genetic and optogenetic tools reveal hippocampal CA2 circuits. Nat. Neurosci. 17, 269-279. 
Leutgeb, J.K., Leutgeb, S., Moser, M.B., Moser, E.I., 2007. Pattern separation in the dentate gyrus and CA3 of the hippocampus. Science 315, 961-966.

Lledo, P.M., Merkle, F.T., Alvarez-Buylla, A., 2008. Origin and function of olfactory bulb interneuron diversity. Trends Neurosci. 31, 392-400.

Llorens-Martin, M., Torres-Aleman, I., Trejo, J.L., 2006. Pronounced individual variation in the response to the stimulatory action of exercise on immature hippocampal neurons. Hippocampus 16, 480-490.

Llorens-Martin, M., Teixeira, C.M., Fuster-Matanzo, A., Jurado-Arjona, J., Borrell, V., Soriano, E., Avila, J., Hernandez, F., 2012. Tau isoform with three microtubule binding domains is a marker of new axons generated from the subgranular zone in the hippocampal dentate gyrus: implications for Alzheimer's disease. J. Alzheimers Dis. 29, 921-930.

Llorens-Martin, M., Fuster-Matanzo, A., Teixeira, C.M., Jurado-Arjona, J., Ulloa, F., Defelipe, J., Rabano, A., Hernandez, F., Soriano, E., Avila, J., 2013. GSK-3beta overexpression causes reversible alterations on postsynaptic densities and dendritic morphology of hippocampal granule neurons in vivo. Mol. Psychiatry 18, 451-460.

Maggio, N., Shavit-Stein, E., Dori, A., Blatt, I., Chapman, J., 2013. Prolonged systemic inflammation persistently modifies synaptic plasticity in the hippocampus: modulation by the stress hormones. Front. Mol. Neurosci. 6, 46.

Mercer, A., Trigg, H.L., Thomson, A.M., 2007. Characterization of neurons in the CA2 subfield of the adult rat hippocampus. J. Neuroscie. 27, 7329-7338.

Monje, M.L., Toda, H., Palmer, T.D., 2003. Inflammatory blockade restores adult hippocampal neurogenesis. Science 302, 1760-1765.
Nakashiba, T., Cushman, J.D., Pelkey, K.A., Renaudineau, S., Buhl, D.L., McHugh, T.J. Rodriguez Barrera, V., Chittajallu, R., Iwamoto, K.S., McBain, C.J., Fanselow, M.S., Tonegawa, S., 2012. Young dentate granule cells mediate pattern separation, whereas old granule cells facilitate pattern completion. Cell 149, 188-201.

Schmidt, B., Marrone, D.F., Markus, E.J., 2012. Disambiguating the similar: the dentate gyrus and pattern separation. Behav. Brain Res. 226, 56-65.

Schmidt-Hieber, C., Jonas, P., Bischofberger, J., 2004. Enhanced synaptic plasticity in newly generated granule cells of the adult hippocampus. Nature 429, 184-187.

Toni, N., Laplagne, D.A., Zhao, C., Lombardi, G., Ribak, C.E., Gage, F.H., Schinder, A.F., 2008 Neurons born in the adult dentate gyrus form functional synapses with target cells. Nat. Neurosci. 11, 901-907.

van Strien, N.M., Cappaert, N.L., Witter, M.P., 2009. The anatomy of memory: an interactive overview of the parahippocampal-hippocampal network. Nat. Rev. Neurosci. $10,272-282$.

Wintzer, M.E., Boehringer, R., Polygalov, D., McHugh, T.J., 2014. The hippocampal CA2 ensemble is sensitive to contextual change. J. Neurosci. 34, 3056-3066.

Zhao, C., Teng, E.M., Summers Jr., R.G., Ming, G.L., Gage, F.H., 2006. Distinct morphological stages of dentate granule neuron maturation in the adult mouse hippocampus. J. Neurosci. 26, 3-11.

Zhao, M., Choi, Y.S., Obrietan, K., Dudek, S.M., 2007. Synaptic plasticity (and the lack thereof) in hippocampal CA2 neurons. J. Neuroscie. 27, 12025-12032. 